Article

\title{
A Transient Fault Recognition Method for an AC-DC Hybrid Transmission System Based on MMC Information Fusion
}

\author{
Jikai Chen *, Yanhui Dou, Yang Li, Jiang Li and Guoqing Li \\ School of Electrical Engineering, Northeast Electric Power University, Jilin 132012, China; \\ fundouyh@163.com (Y.D.); liyang@nedu.edu.cn (Y.L.); lijiangdyx@163.com (J.L.); lgq@nedu.edu.cn (G.L.) \\ * Correspondence: chenjikai1977@163.com; Tel.: +86-432-6480-6691
}

Academic Editor: Ying-Yi Hong

Received: 29 August 2016; Accepted: 19 December 2016; Published: 26 December 2016

\begin{abstract}
At present, the research is still in the primary stage in the process of fault disturbance energy transfer in the multilevel modular converter based high voltage direct current (HVDC-MMC). An urgent problem is how to extract and analyze the fault features hidden in MMC electrical information in further studies on the HVDC system. Aiming at the above, this article analyzes the influence of AC transient disturbance on electrical signals of MMC. At the same time, it is found that the energy distribution of electrical signals in MMC is different for different arms in the same frequency bands after the discrete wavelet packet transformation (DWPT). Renyi wavelet packet energy entropy (RWPEE) and Renyi wavelet packet time entropy (RWPTE) are proposed and applied to AC transient fault feature extraction from electrical signals in MMC. Using the feature extraction results of Renyi wavelet packet entropy (RWPE), a novel recognition method is put forward to recognize AC transient faults using the information fusion technology. Theoretical analysis and experimental results show that the proposed method is available to recognize transient AC faults.
\end{abstract}

Keywords: transient faults; MMC; discrete wavelet packet transformation; Renyi entropy; feature extraction; fault recognition

\section{Introduction}

Conventional HVDC usually adopts line commuted converter high voltage direct current (LCC-HVDC) technology. Power transmission of LCC-HVDC system is based on thyristor. However, thyristor does not have the capacity to turn off itself, and it has low switching frequency, which causes low performance for converters. In an LCC-HVDC system, receive port must be a high-capacity power system as to provides commutation current. Only in this way can power be transmitted to the grid. Because thyristor does not have the capacity of inverter commutation by itself, and LCC-HVDC system does not work when receive port is a low-capacity power system or commutation current is not fully provided. MMC-HVDC is developed on the basis of conventional HVDC, so it has the advantages of conventional HVDC [1]. MMC based on insulated gate bipolar transistor (IGBT) is a new technology that is widely used in voltage-source converters [2,3]. As a new HVDC technology, MMC-HVDC introduces fully controlled power electronics devices and PWM technology, and solves many problems that exist in conventional HVDC [4-8]. Large amounts of researches have been conducted on MMC-HVDC, so it widely applies in many areas such as the large-capacity power delivery, asynchronous networks and island power transmission [9-12]. According to the topology structure of MMC, reference [13] analyzes fault characteristics of typical transient faults, and the electromagnetic transient model of AC side of MMC is derived under the condition of unbalance AC grid. The control strategies are designed to reduce current circulation in MMC. In reference [14], 
the structure and principle of MMC are discussed. Then, the internal bus faults are reviewed, and a protection strategy focusing on single-phase ground fault is proposed. In reference [15], the positive sequence and negative sequence components of $\mathrm{AC}$ are separated, and the mathematical model of MMC-HVDC system is established. Then its fault features under three-phase unbalance fault are analyzed, which include the changing processes of AC, DC, SM capacitance voltage, and the internal current, and a protection strategy is also proposed. In references [16-18], the structure and principle of MMC are discussed. Then, characteristic of faults are analyzed, and the corresponding protection strategy is proposed.

Although a number of researches have been conducted, the research is still in initial stage on process of evaluation of operation state and AC-DC interaction for MMC-HVDC system. Meanwhile, the in-depth researches are also needed to improve the extraction, induction and analysis technologies of the fault characteristics hidden in MMC electrical information. For the above, on basis of Renyi wavelet packet energy entropy (RWPEE) and Renyi wavelet packet time entropy (RWPTE) proposed, a transient feature extraction method is proposed and applied to the recognition of transient faults on AC side of MMC. This method provides theoretical supportfor theevaluation of operation state and AC-DC interaction for MMC-HVDC system. The theoretical analysis and experimental results prove the effectiveness of the proposed method.

\section{Influence of AC Transient Faults on MMC}

A MMC consists of three-phase six-arm circuits, in which each arm is united by several sub-modules (SM), the equivalent inductance is $L_{0}$ and the equivalent resistance is $R_{0}$ in series. Upper and lower arms compose a phase unit, as shown in Figure 1.

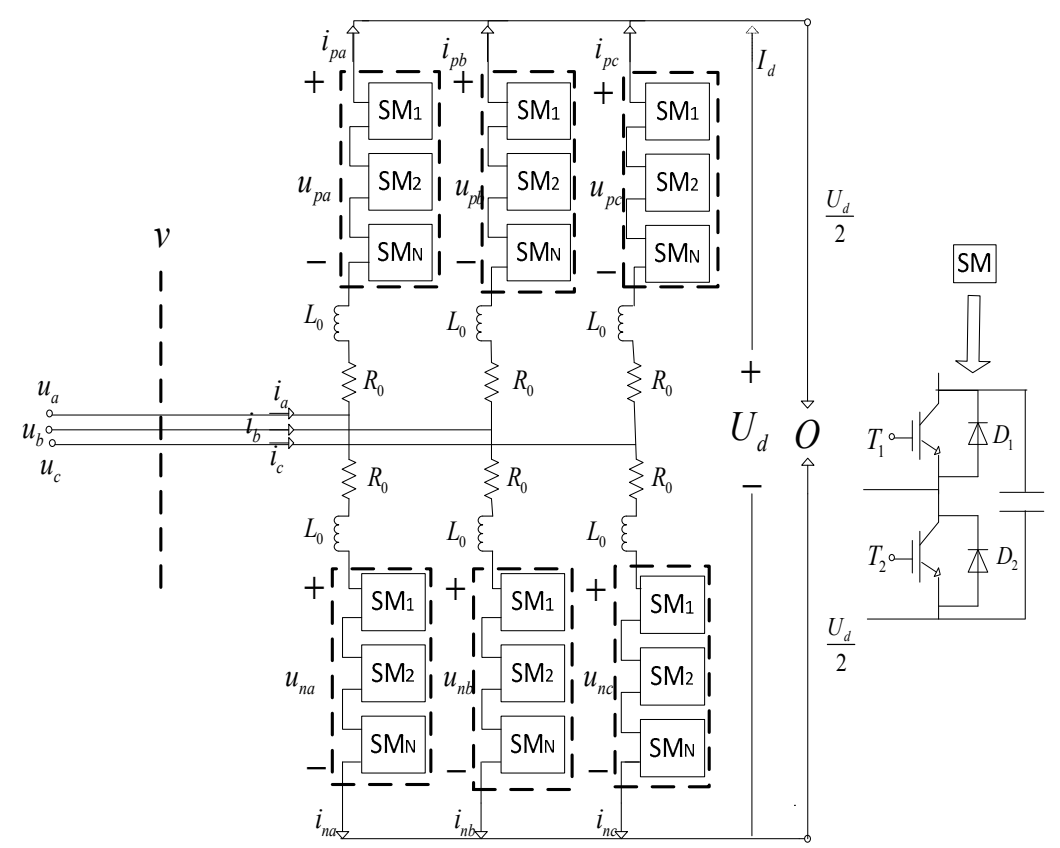

Figure 1. Equivalent circuit of a multilevel modular converter (MMC).

In each SM, $T_{1}$ and $T_{2}$ can be controlled with "on" and "off" to change the output. By controlling the SM's of upper and lower arms, multilevel waveform is output to AC side of MMC.

The point $v$ is AC input of the converter, which can connect with AC system by transformer. The voltage and input current of $v$ is $u_{j}$ and $i_{j}(j=a, b, c)$ respectively. $U_{d c}$ is DC voltage and $I_{d}$ is DC current. The arm voltages of six controlled voltage sources are $u_{p j}$ and $u_{n j}(j=a, b, c)$. At the same time, the arm currents are $i_{p j}$ and $i_{n j}(j=a, b, c)$. Among them, the subscripts $p$ and $n$ represent upper arm and lower arm respectively, and $o$ is the neutral point. Reference direction is as shown in Figure 1 [19]. 
As shown in Figure 1, according to Kirchhoff current theory,

$$
i_{j}=i_{p j}+i_{n j}, j=a, b, c
$$

The current in the arms in the $j$-th phase unit is regarded as [20],

$$
i_{\text {jdif }}=\frac{i_{p j}-i_{n j}}{2}
$$

$I_{j d c}$ and $i_{j a c}$ represent DC component and AC component in the current of arms. Therefore, the upper and lower arm currents in $j$ phase are regarded as,

$$
\begin{aligned}
& i_{p j}=\frac{i_{j}}{2}+I_{j d c}+i_{j a c} \\
& i_{n j}=\frac{i_{j}}{2}-I_{j d c}-i_{j a c}
\end{aligned}
$$

According to Kirchhoff current theory, the equilibrium equations for upper and lower arms in $j$ phase are regarded as [21]

$$
\begin{gathered}
u_{j}-\left(\frac{U_{d}}{2}-u_{p j}\right)=L_{0} \frac{d i_{p j}}{d t}+R_{0} i_{p j} \\
u_{j}-\left(-\frac{U_{d}}{2}+u_{n j}\right)=L_{0} \frac{d i_{n j}}{d t}+R_{0} i_{n j}
\end{gathered}
$$

Adding Equations (5) to (6), it is derived as

$$
u_{j}-\frac{u_{n j}-u_{p j}}{2}=L_{0} \frac{d i_{j}}{d t}+R_{0} i_{j}
$$

Therefore, Equation (7) can also be rewritten as the form of three-phase system

$$
\left[\begin{array}{l}
u_{n a}-u_{p a} \\
u_{n b}-u_{p b} \\
u_{n c}-u_{p c}
\end{array}\right]=2\left[\begin{array}{l}
u_{a} \\
u_{b} \\
u_{c}
\end{array}\right]-2 R_{0}\left[\begin{array}{c}
i_{a} \\
i_{b} \\
i_{c}
\end{array}\right]-2 L_{0} \frac{d}{d t}\left[\begin{array}{c}
i_{a} \\
i_{b} \\
i_{c}
\end{array}\right]
$$

Equation (8) describes the dynamic characteristics of AC side in MMC. Equation (6) is subtracted from Equation (5), it is obtained as

$$
U_{d}=\left(u_{p j}+u_{n j}\right)-2 R_{0} I_{j d c}-2 R_{0} i_{j a c}-2 L_{0} \frac{d i_{j a c}}{d t}
$$

The current on DC side can be obtained as

$$
I_{d}=\sum_{j=a, b, c}\left(I_{j d c}+i_{j a c}\right)
$$

Equations (9) and (10) describe the dynamic characteristics of DC side in MMC. Dynamic mathematical model of MMC by using Kirchhoff theory is applied not only in normal conditions but also in fault conditions. When transient fault occurs on AC transmission line, the internal arm voltages $u_{p j}, u_{n j}(j=a, b, c)$ will change because of the fluctuation of $u_{j}(j=a, b, c)$. The voltages of arm will be adjusted by control strategy of MMC, which leads to the fluctuation of reactive power and the change of $i_{p j}, i_{n j}(j=a, b, c)$, eventually the fluctuation of $U_{d}$ and $I_{d}$ on DC bus. In order to verify the theoretical analysis above, simulation model, 2-terminal 51-level MMC station, is built on RT-LAB (11.0) and MATLAB (2013a) simulation platform. As AB-phase ungrounded short circuit fault occurs on AC transmission line, the original electrical signals are as shown in Figures 2 and 3. From Figures 2 and 3 , 
it can be found that the voltage and current of arm and DC bus fluctuate significantly when the fault occurs.

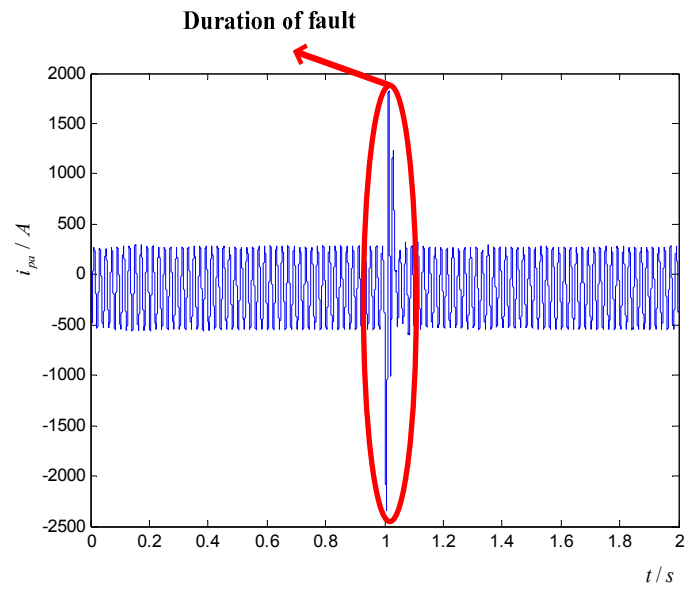

(a)

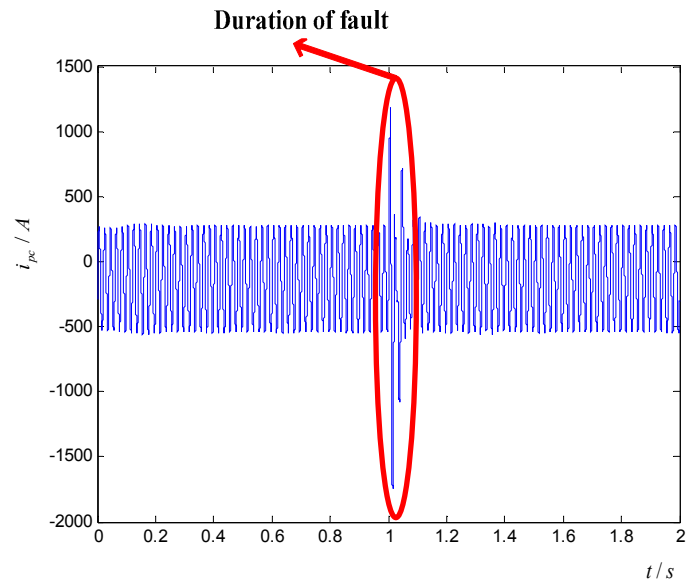

(c)

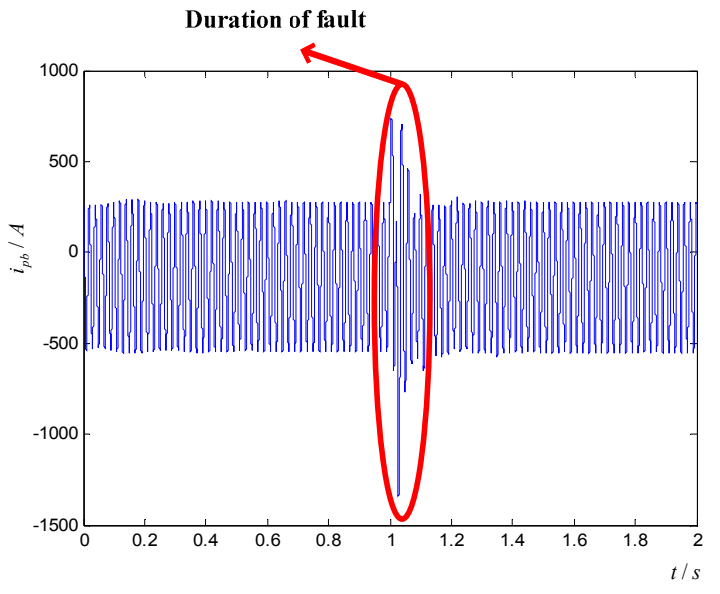

(b)

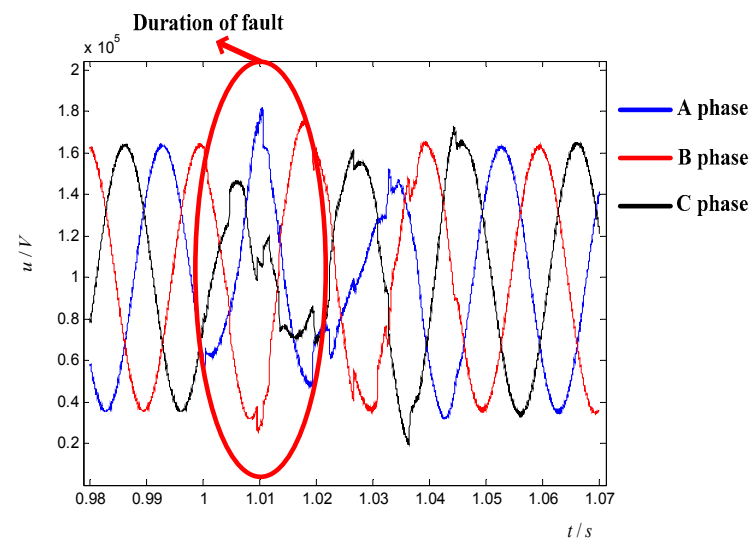

(d)

Figure 2. The upper arm signals when AB-phase ungrounded short circuit fault occurs. (a) A-phase current; (b) B-phase current; (c) C-phase current; (d) ABC-phase voltage.

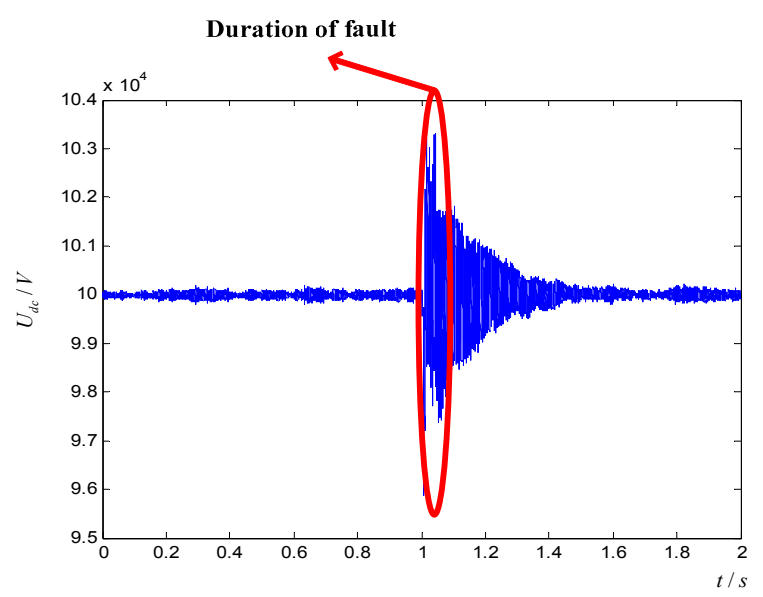

Figure 3. The voltage of DC bus when AB-phase ungrounded short circuit fault occurs. 


\section{Time Frequency Analysis of Arm Current under Transient Fault Condition}

According to the above analysis of the influence of AC transient faults on MMC, the voltage and current of arms and DC bus in MMC will be changed when AC transient faults occur on AC transmission line. However, because of the system damping, circulating-current restrain and control strategy, it is difficult to determine the AC fault types and fault phases directly according to the change of time-domain current and voltage in MMC.

Based on the MMC-HVDC model, arm currents in MMC can be obtained by simulating AB-phase grounding short circuit fault. By comparison of Figures 2 and 4, there are not the obvious differences in the fault current between $A B$-phase grounding short circuit fault and AB-phase ungrounded short circuit fault. It is difficult to determine fault types and fault phases directly only according to the current waveform in time domain.

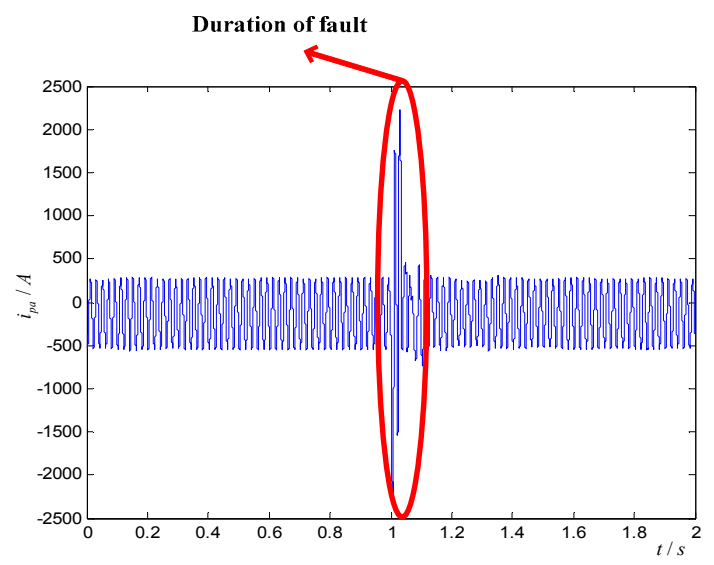

(a)

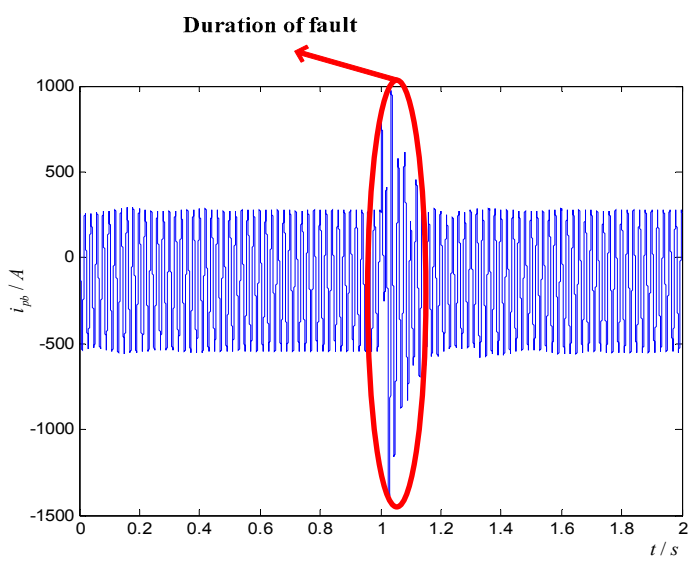

(b)

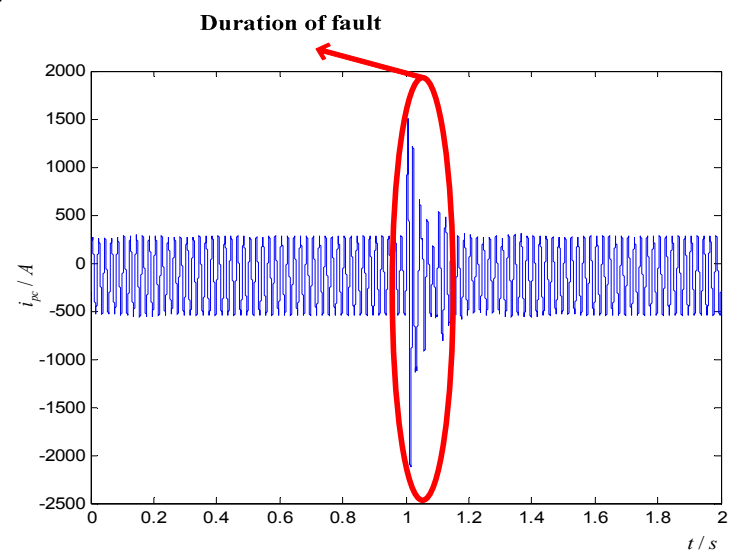

(c)

Figure 4. The upper arm signals when AB-phase grounding short circuit fault occurs. (a) A-phase current; (b) B-phase current; (c) C-phase current.

DWPT can capture the local information in time-frequency domain. At the same time, it is an optical choice for analysis and detection on transient signals and non-stationary signals [22-24]. Based on the above analysis, when AB-phase ungrounded short circuit fault occurs, the arm currents in MMC is taken as an example, and it is split into different frequency components with DWPT in 0-112 Hz. Through analyzing, there are significant differences in the energy distribution of three-phase arm currents in $14-28 \mathrm{~Hz}$ when the fault occurs on AC transmission line, as shown in Figure 5. 


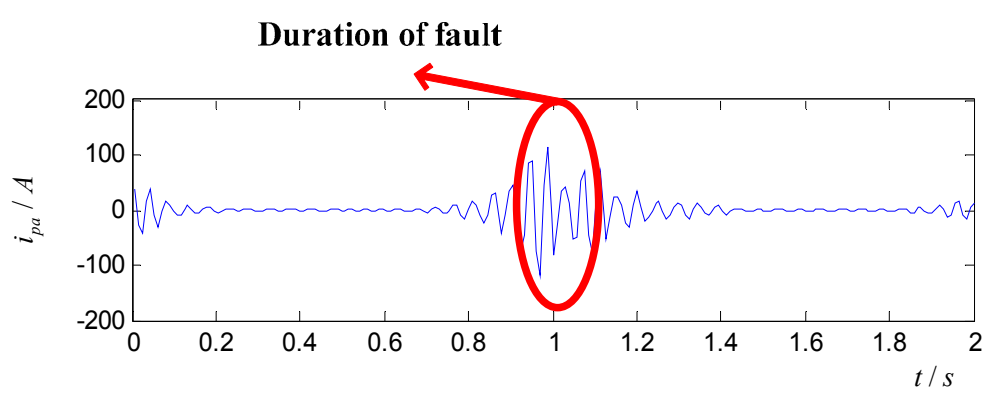

(a)

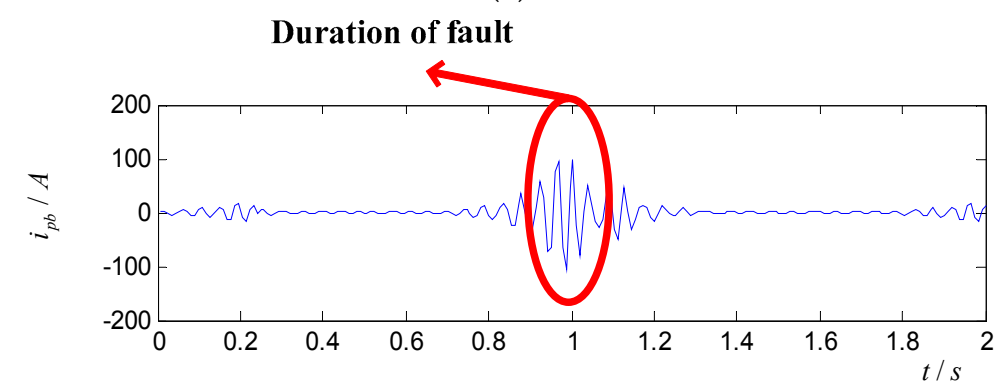

(b)

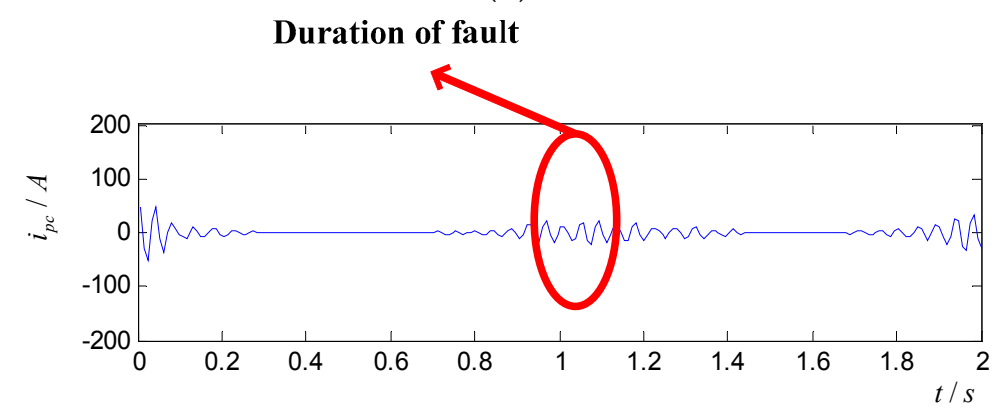

(c)

Figure 5. The upper arm current when $A B-p h a s e$ grounding short circuit fault occurs $(14-28 \mathrm{~Hz})$.

(a) A-phase current; (b) B-phase current; (c) C-phase current.

From Figure 5, we can see that there is a significant change of current in AB-phase when fault occurs, but slight fluctuations in C-phase. The further work reveals that the energy distribution is different among different phases and frequency bands when different transient faults occur on AC transmission line. Only by using DWPT to obtain the information in each band, the amount of information is huge and the fault features are not significant because of the complexity of transient signals in different phases and frequency bands. Therefore, it is unable to exactly identify the type and phase of fault. In the basis of the multi-resolution of DWPT, a novel method of transient signal analysis can be proposed combining with entropy statistics. The further data mining can be performed to make the fault features easy to recognize with the proposed method.

\section{Feature Extraction of AC Transient Faults Based on Renyi Wavelet Packet Entropy}

\subsection{The Definition of Renyi Entropy}

Renyi entropy is an extension of Shannon entropy, Renyi entropy is equivalent to Shannon when $\alpha=1$. Because of the adjustable parameters $\alpha$ introduced by Renyi entropy, the parameter $\alpha$ can be adjusted according to the signal feature from the prior knowledge. Renyi entropy is defined as follows [25]. 


$$
W_{R E}(m)=\left\{\begin{array}{cc}
\frac{1}{1-\alpha} \ln \left(\sum_{n} p_{m}^{\alpha}(j)\right) & \alpha>0, \alpha \neq 1 \\
-\sum_{n} p_{m}(j) \ln p_{m}(j) & \alpha=1
\end{array}\right.
$$

\subsection{The Statistical Properties of Renyi Entropy}

The selection of $\alpha$ plays an important role in the statistical properties of Renyi entropy. Taking a three-level system as the analysis object, according to Equation (11), the statistical results are calculated, and the corresponding relation between Renyi entropy with $\alpha$ and the probability distribution are shown in Figure 6. According to Figure 6, when $\alpha>0$ and $\alpha \neq 0$, with the increase of $\alpha$, the statistical range of Renyi entropy will expand for the system state of small probability event, and the statistical sensitivity for the small probability event will reduce correspondingly. In contrast, with the decrease of $\alpha$, the statistical range of small probability event is reduced, and the statistical sensitivity increases [26].

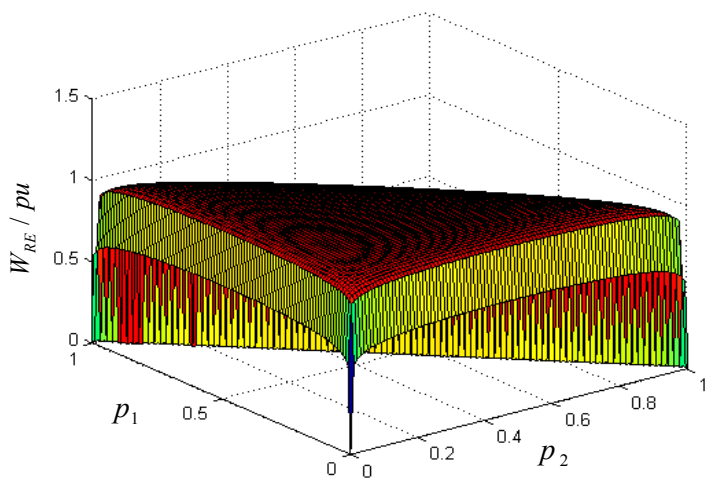

(a)

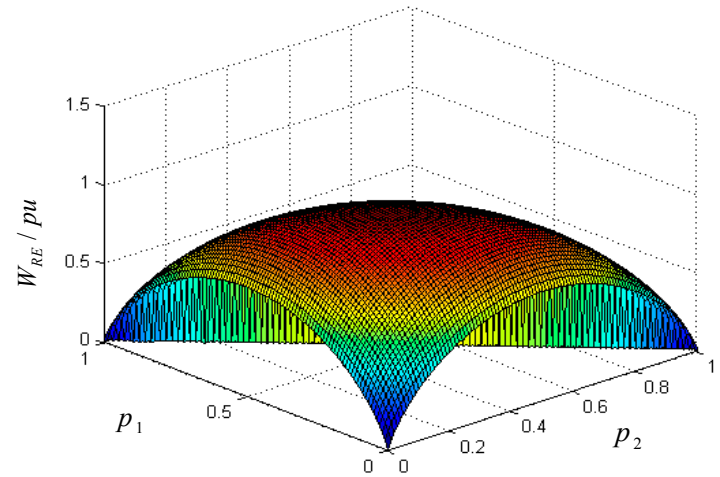

(c)

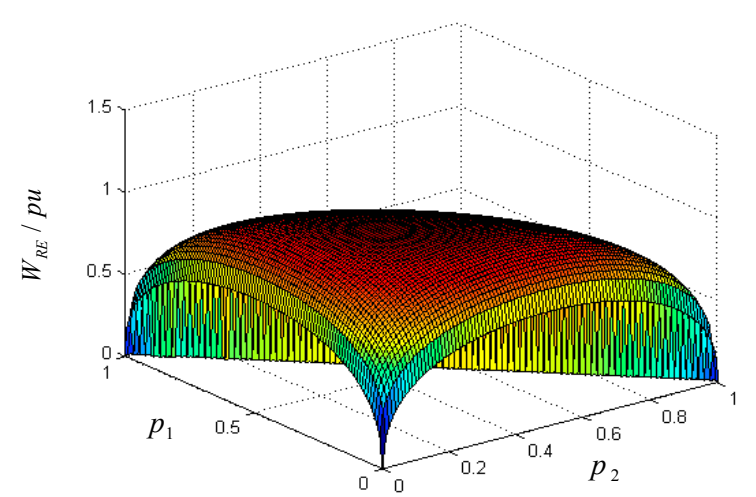

(b)

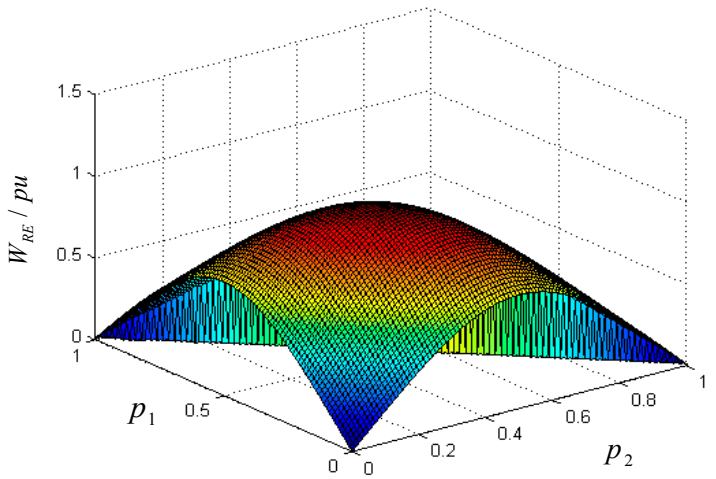

(d)

Figure 6. Relation between Renyi entropy with different values of $\alpha$ and probability distribution. (a) $\alpha=0.1$; (b) $\alpha=0.5 ;$ (c) $\alpha=0.99 ;$ (d) $\alpha=2$.

For a signal containing transient components, the components of the signal are considered as the small probability event. Therefore, Renyi entropy can get better results in extracting the signal feature with the appropriate $\alpha$, and $\alpha$ should be smaller than 0.5. Based on principles above, $\alpha$ should be limited in 0.1-0.3 according to the energy distribution of measured signal in each frequency band.

\subsection{Renyi Wavelet Packet Energy Entropy}

DWPT can get much richer time-frequency local information. It is better applied to analyze and detect the saltation of signals. Therefore, DWPT is introduced. The DWPT is listed as follows $[27,28]$. 


$$
\left\{\begin{array}{l}
d_{i, 2 k}(t)=\sqrt{2} \sum_{k} g(x) d_{i-1, k}(2 t-x) \\
d_{i, 2 k-1}(t)=\sqrt{2} \sum_{k} h(x) d_{i-1, k}(2 t-x) \\
d_{0,0}(t)=x(t)
\end{array}\right.
$$

where $h(x)$ is a high-pass filter, $g(x)$ is a low-pass filter, $d_{i, k}(x)$ is the reconstructed signal of the node $k$ on the layer $i$ by DWPT, DWPT is to divide each sub-band into two parts and transmit them to the lower levels until each sub-band corresponds to a frequency component of signal.

Decomposing signal into $i$ layers. Then, the node coefficient of discrete wavelet packet or reconstructed signals are obtained to build the matrix $D_{1}=\left\{d_{i, j}(k), k=1, \ldots, L, 1 \leq i \leq N\right.$, $\left.j=1, \ldots, 2^{i}\right\}, L$ is the length of $x(n)$. A sliding window is defined as $W(m, w, \delta)$ on $D_{L \times S}, w$ is the window width, $\delta$ is the sliding factor. This sliding window is shown as follows [29],

$$
W(m, w, \delta)=\left(\begin{array}{cccc}
d_{i, 1}(1+m \delta) & d_{i, 1}(2+m \delta) & \cdots & d_{i, 1}(w+m \delta) \\
d_{i, 2}(1+m \delta) & d_{i, 2}(2+m \delta) & \cdots & d_{i, 2}(w+m \delta) \\
\vdots & \vdots & \vdots & \vdots \\
d_{i, 2^{i}}(1+m \delta) & d_{i, 2^{i}}(2+m \delta) & \cdots & d_{i, 2^{i}}(w+m \delta)
\end{array}\right)
$$

In the above equation, $m=1,2, \ldots, M, M=(L-w) / \delta, d_{i, j}(k)$ is the $k$-th node coefficient or reconstructed signal of wavelet packet nodes $(i, j) . k$ is the position variables of node coefficient or reconstructed signal, $N$ is the upper limit of decomposition level, $M$ is the length of RWPEE. The operation process of RWPEE is listed as follows.

$E(m)=\sum_{j=1}^{2^{i}} E_{m}(j)$ is the energy sum of $2^{i}$ wavelet packet coefficient set or reconstructed signal energy. The wavelet packet coefficient set and reconstructed signal energy are on scale $i$ of the sliding window which is centered on the time $(m+w / 2)$ and with a window width of $w \in N$. $E_{m}(j)=\sum_{k=1+m \delta}^{w+m \delta}\left(d_{i, j}(k)\right)^{2}$ is the energy sum of the $j$-th wavelet packet coefficient set or reconstructed signal energy. The wavelet packet coefficient set and reconstructed signal energy are on scale $i$ of the sliding window which is centered on the time $(m+w / 2)$ and with a window width of $w \in N$.

When $p_{m}(j)=E_{m}(j) / E(m)$ and $\sum_{j=1}^{2^{i}} p_{m}(j)=1$, RWPEE at $(m+w / 2)$ is shown as follow,

$$
W_{\text {RWPEE }}(m)=\frac{1}{1-\alpha} \ln \left(\sum_{j=1}^{2^{i}}\left(p_{m}(j)\right)^{\alpha}\right)
$$

\subsection{Renyi Wavelet Packet Time Entropy}

Decomposing signal into $i$ layers. Then, the node coefficient of discrete wavelet packet or reconstructed signals are obtained to build the matrix $D_{2}=\left\{d_{i, j}(k), k=1, \ldots, L, 1 \leq i \leq N, j=1, \ldots, 2^{i}\right\}$, $L$ is the length of $x(n)$. A sliding window is defined on the $j$-th wavelet packet coefficient set or reconstructed signal energy, $w$ is the window width, $\delta$ is the sliding factor. This sliding window is shown as follows,

$$
W(m, w, \delta)=\left(d_{i, j}(1+m \delta), \ldots, d_{i, j}(w+m \delta)\right)
$$

In the above equation, $m=1,2, \ldots, M, M=(L-w) / \delta$. We break up the $D_{2}$ into $R$ intervals,

$$
D_{2}=\bigcup_{l=1}^{R} Z_{l}
$$

In this equation, $\left\{Z_{l}=\left[s_{l-1}, s_{l}\right), l=1,2, \ldots, R\right\}, s_{0}<s_{1} \ldots<s_{R}, s_{0}=\min \left(D_{2}\right)$ and $s_{R}=\max \left(D_{2}\right)$. 
Let $p_{m}\left(Z_{l}\right)$ as the wavelet coefficients (reconstruction signal) $d_{i}(k)$ probability which dropping into interval $Z_{l}$. The probability equals to the ratio of the number of the wavelet coefficients (reconstruction signal) $d_{i}(k)$ which dropping into interval $Z_{l}$ to the total number of which in the sliding window $W(m, w, \delta)$. Therefore, RWPTE at $(m+w / 2)$ in $j$-th scale is shown as,

$$
W_{\operatorname{RWPTE}}(m)=\frac{1}{1-\alpha} \ln \left(\sum_{l=1}^{R}\left(p_{m}\left(Z_{l}\right)\right)^{\alpha}\right)
$$

\subsection{ContrastAnalysisof RWPE and SWPE}

Although Shannon wavelet packet entropy (SWPE) is widely applied in a power system, when the measured signal is more complex and contains a lot of random signals, there is severe energy leakage and frequency aliasing in the wavelet coefficients (or reconstructed signals) with increasing of the wavelet decomposition scale. It reduces accuracy of feature extraction and could cause that feature extraction is submerged in the background noise [30]. Due to adjustable parameter $\alpha$ of Renyi, based on the feature of measured signals, $\alpha$ is adjusted to reduce the effect of wavelet aliasing on accuracy of feature extraction. Based on the above analysis, A-phase grounded short circuit fault on AC transmission line is taken as an example to verify the theoretical analysis. Here, the fault occurs at $1 \mathrm{~s}$. The RWPEE proposed and Shannon wavelet packet energy entropy (SWPEE) are used to extract fault feature from DC bus voltage. The results of feature extraction are shown in Figure 7. By analyzing Figure 7, RWPEE can extract effectively feature of transient fault, and the accuracy of feature extraction is better than SWPEE. Then, feature extraction results of RWPTE also are superior to Shannon wavelet packet time entropy (SWPTE). Therefore, comparing SWPE, RWPE proposed have high accuracy in feature extraction of AC transient faults.

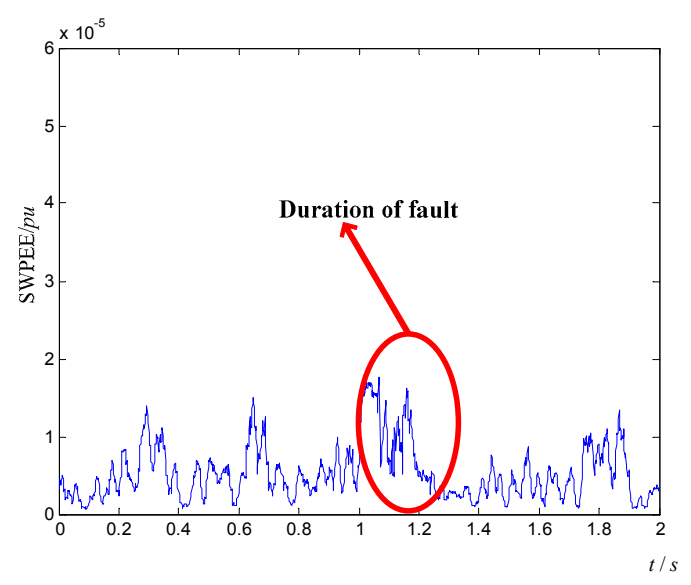

(a)

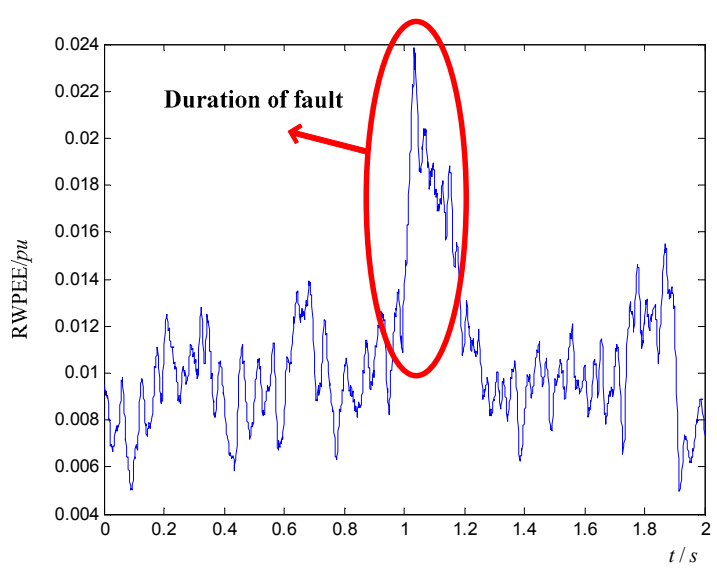

(b)

Figure 7. Feature extraction of fault feature from DC bus voltage. (a) SWPEE; (b) RWPEE.

In term of computation complexity, complexity of RWPE algorithm is affected by computation of Mallat wavelet transform and Renyi entropy. RWPE is as an example to analyze complexity of RWPE algorithm based on Db4 wavelet transform. Mallat algorithm is that discrete wavelet is transformed into high-pass and low-pass filters to repeatedly filter for signals. Coefficients of low-pass filters with Db4 wavelet transform are known. According to the mathematical relationship (Equation (18)) between decomposition filters and reconstruction filters, coefficients of high-pass filters are acquired.

$$
\left\{\begin{array}{l}
H(n)=h(-n) \\
G(n)=g(-n) \\
g(n)=(-1)^{1-n} h(1-n)
\end{array}\right.
$$


$H(n)$ is low-pass decomposition filter, $G(n)$ is high-pass decomposition filter, $h(n)$ is low-pass reconstruction filter, $g(n)$ is high-pass reconstruction filter. Therefore, $z$-transform of filter banks is expressed as follows.

$$
\left\{\begin{array}{l}
G(z)=-0.2304 z^{-1}+0.7148-0.6309 z-0.0280 z^{2}+0.1870 z^{3}+0.0308 z^{4}-0.0329 z^{5}-0.0106 z^{6} \\
H(z)=0.0106 z^{-7}+0.0329 z^{-6}+0.0308 z^{-5}-0.10870 z^{-4}-0.0280 z^{-3}+0.6309 z^{-2}+0.7148 z^{-1}+0.2034
\end{array}\right.
$$

where, $G(z)$ is z-transform of high-pass filter, $H(z)$ is z-transform of low-pass filter. For convenience, Equation (19) is transformed into recurrence relation as follows.

$$
\left\{\begin{aligned}
d(n)= & -0.2304 x(n-1)+0.7148 x(n)-0.6309 x(n+1)-0.0280 x(n+2) \\
& +0.1870 x(n+3)+0.0308 x(n+4)-0.0329 x(n+5)-0.0106 x(n+6) \\
a(n)= & 0.0106 x(n-7)+0.0329 x(n-6)+0.0308 x(n-5)-0.10870 x(n-4) \\
& -0.0280 x(n-3)+0.6309 x(n-2)+0.7148 x(n-1)+0.2034 x(n)
\end{aligned}\right.
$$

where $x(n)$ is time series of measured signal. Based on Equation (20), Db4 wavelet decomposition needs $16 M+14 A$ operations in a single computation ( $M$ is multiplication, and $A$ is addition). If a branch signal is decomposed into the reconstructed signals, the computation of the branch signal will be doubled. According to the Renyi entropy equation, it is necessary to compute a Renyi entropy with $N_{x}$ $(A+P)+M+Q$ operations ( $Q$ is logarithm and $P$ is exponentiation) when the numbers of probability are $N_{x}$. According to the above analysis, based on Db4 wavelet basis function, RWPE with operation complexity are shown in Table 1. Compared with the SWPE algorithm that is widely applied to feature extraction of transient faults, RWPE is superior to SWPE in complexity.

Table 1. Complexity of algorithm.

\begin{tabular}{clll}
\hline Entropy & Complexity of RWPE Algorithm & Complexity of SWPE Algorithm & Computational Cost Savings \\
\hline \multirow{2}{*}{ Time Entropy } & $L(16 M+14 A) \sum_{i=1}^{S}\left(2^{-i}\right)+$ & $L(16 M+14 A) \sum_{i=1}^{S}\left(2^{-i}\right)+$ & $\frac{(L-w)}{\delta}(R(M-1)-Q+R J)$ \\
& $\frac{(L-w)}{\delta}\left(R(A+P)+M+Q+M_{T}\right)$ & $\frac{(L-w)}{\delta}\left(R(A+M+Q+J)+M_{T}\right)$ & \\
\hline \multirow{2}{*}{ Energy Entropy } & $L(16 M+14 A) \sum_{i=1}^{S}\left(2^{-i}\right)+$ & $L(16 M+14 A) \sum_{i=1}^{S}\left(2^{-i}\right)+$ & $\frac{(L-w)}{\delta}(S(M-1)-Q+S J)$ \\
& $\frac{(L-w)}{\delta}\left(S(A+P)+M+Q+M_{E}\right)$ & $\frac{(L-w)}{\delta}\left(S(A+M+Q+J)+M_{E}\right)$ & \\
\hline
\end{tabular}

$w$ is the width of sliding window, $L$ is the length of original data, $\delta$ is the slide factor. $M_{T}$ is the operation of time matrix, $M_{E}$ is the operation of energy matrix, $R$ is the number of data interval, $S$ is the number of wavelet scale, $J$ is the logic judgment of Shannon entropy.

\subsection{Feature Extraction of Transient Faults with RWPEE}

According to the MMC-HVDC model proposed in the Section 6, short circuit faults are be set at $30 \mathrm{~km}$ from the generator, which include A-phase grounding fault, AB-phase grounding short circuit fault, AB-phase ungrounded short circuit fault and three-phase short circuit fault. Then, RWPEE and RWPTE are used to extract the features of transient disturbances hidden in MMC electrical information. Here, the fault occurs at $1 \mathrm{~s}$ and the duration is $0.02 \mathrm{~s}$, data acquisition time is $2 \mathrm{~s}, \alpha$ is 0.1 and grounding resistance is $10 \Omega$. It is found that the variation tendency of currents and voltages in each frequency band are similar in upper and lower arms of MMC when transient disturbance occurs on $\mathrm{AC}$ transmission line. In order to reduce computation and improve the operation efficiency, the upper arm currents and DC bus voltage are selected as the initial data.

The DC bus voltage of A-phase grounding fault, AB-phase grounding short circuit fault, AB-phase ungrounded short circuit fault and three-phase short circuit fault are respectively chosen, where the sampling frequency is $800 \mathrm{~Hz}$. Then, reconstruction signals are computed by RWPEE, the operation results are shown in Figure 8. From Figure 8, when the multi-phase faults occur on AC transmission line, the value of RWPEE is 8-14 times more than that in other time. When A-phase grounding fault occurs, it is about 1.7 times more than that in other time. 


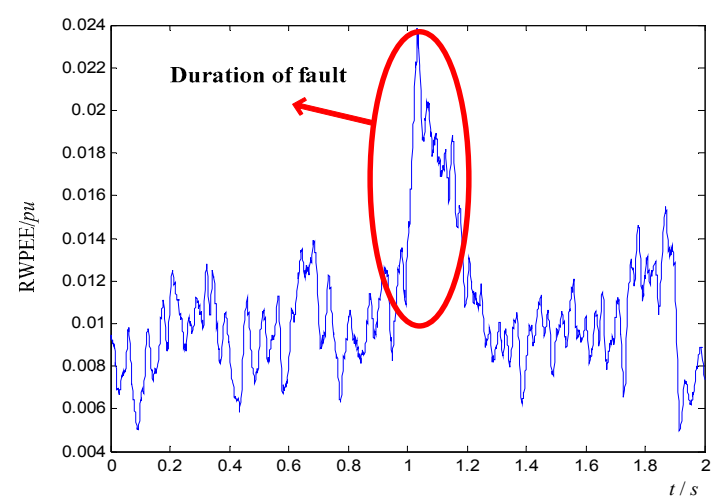

(a)

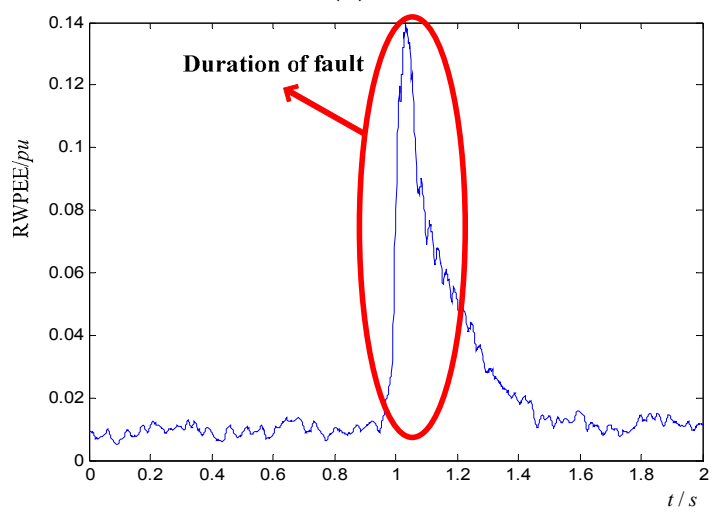

(c)

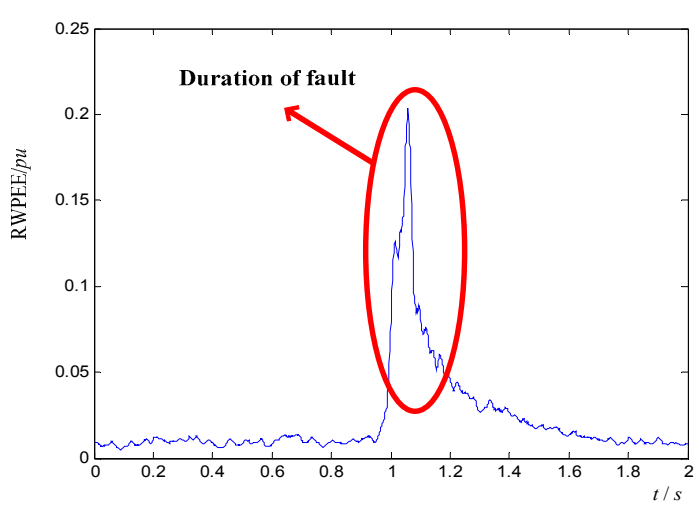

(b)

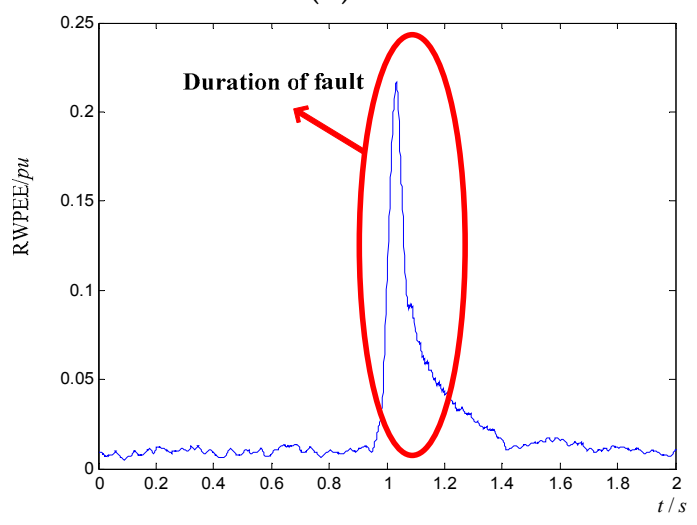

(d)

Figure 8. RWPEE when short circuit fault occurs. (a) A-phase grounding fault; (b) AB-phase grounding short circuit fault; (c) AB-phase ungrounded short circuit fault; (d) ABC-phase short circuit fault.

\subsection{Feature Extraction of Transient Faults with RWPTE}

According to the frequency-domain analysis of arm currents under transient fault condition, when the different transient faults occur on AC transmission line, the energy distribution is different in each frequency band among three phases. Accordingly, RWPTE can be used to extract the feature of transient component in each phase and each frequency band.

When A-phase grounding fault occurs on the AC transmission line, the upper arm currents are respectively sampled with $112 \mathrm{~Hz}$ on MMC. Then, reconstruction signal is computed in $42-49 \mathrm{~Hz}$ by RWPTE, the operation results are shown in Figure 9. In Figure 9, there are no any significant changes in RWPTE of A-phase, but the change occurs in B-phase and C-phase as shown in Figure 8.

When three-phase short circuit fault occurs on the AC transmission line, the upper arm currents are respectively sampled with $112 \mathrm{~Hz}$ on MMC. Then, reconstruction signal is computed in $14-28 \mathrm{~Hz}$ by RWPTE, and the results are shown in Figure 10. As seen in Figure 10, the significant change occurs in the RWPTE of ABC-phase.

When AB-phase grounding short circuit fault occurs on the AC transmission line, the upper arm currents are respectively sampled with $112 \mathrm{~Hz}$. Then, reconstruction signal is computed in $14-28 \mathrm{~Hz}$ by RWPTE, and the results are shown in Figure 11a-c. As seen in Figure 11a-c, there are no any significant changes in RWPTE of C-phase, but the change occurs in A-phase and B-phase.

When AB-phase ungrounded short circuit fault occurs on the AC transmission line, reconstruction signal is computed in 14-28 Hz by RWPTE, and the operation results are shown in Figure 12a-c. In Figure 12a-c, there are no any significant changes in RWPTE of C-phase, but it occurs in A-phase and B-phase. 


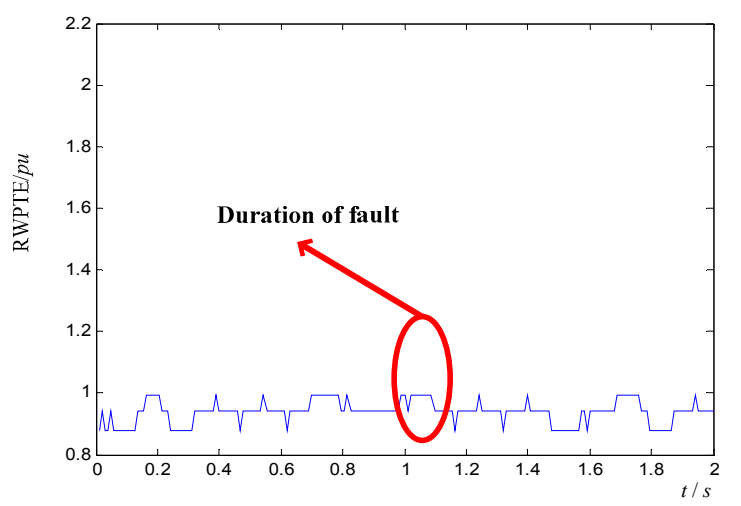

(a)

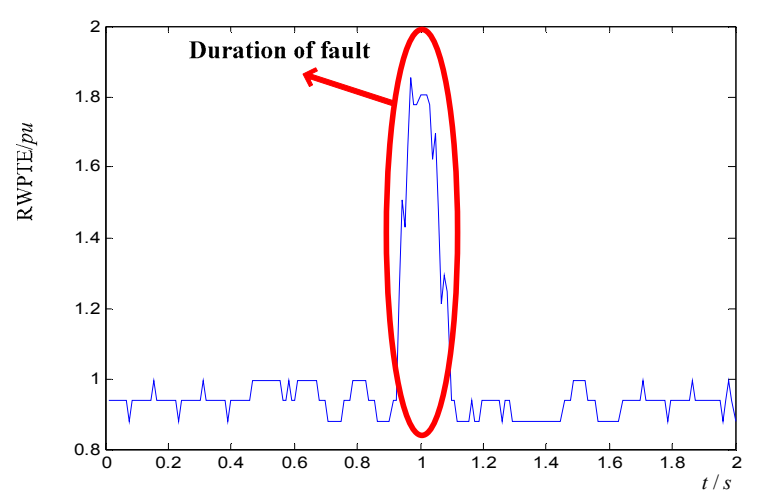

(b)

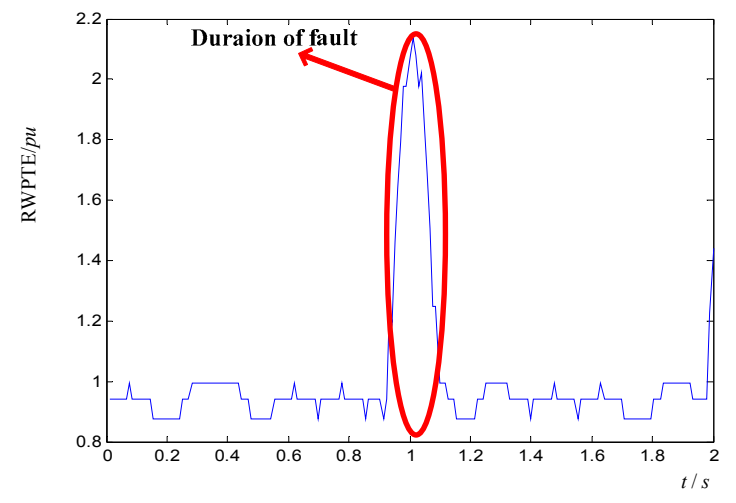

(c)

Figure 9. RWPTE when A-phase grounding fault occurs. (a) A-phase; (b) B-phase; (c) C-phase.

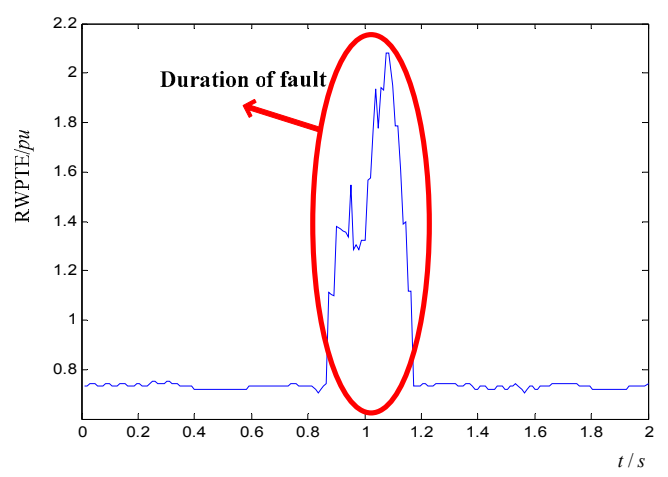

(a)

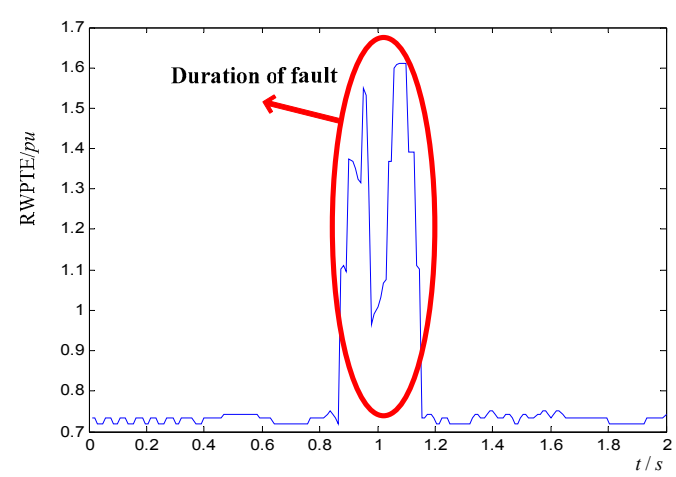

(b)

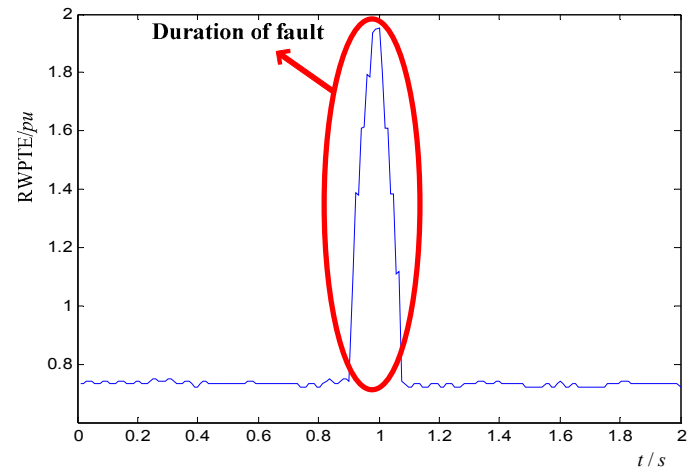

(c)

Figure 10. RWPTE when three-phase short circuit fault occurs. (a) A-phase; (b) B-phase; (c) C-phase. 


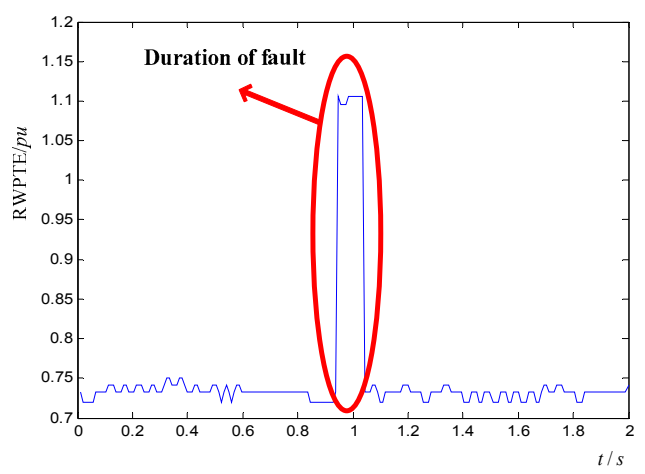

(a)

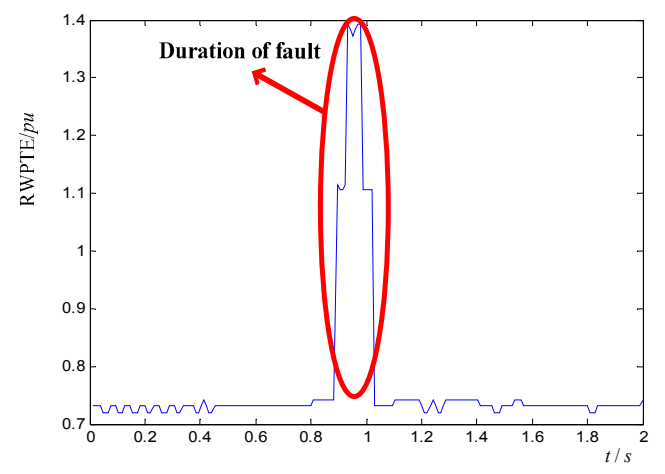

(b)

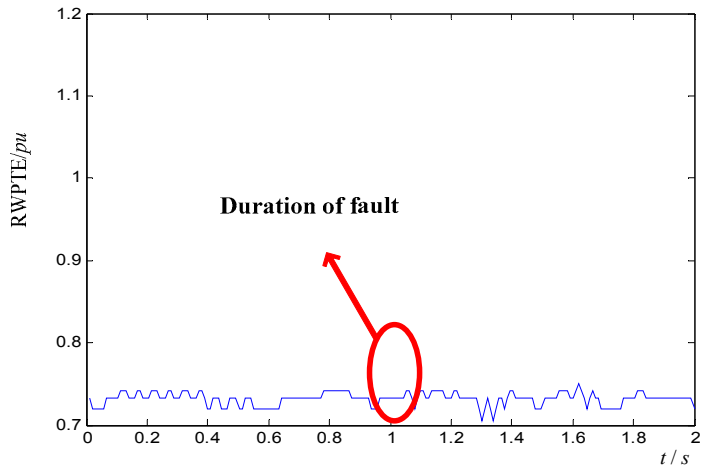

(c)

Figure 11. RWPTE when AB-phase grounding short circuit fault occurs. (a) A-phase; (b) B-phase; (c) C-phase.

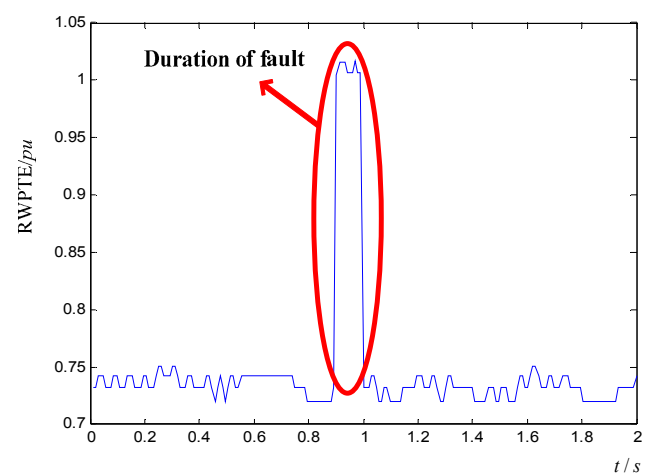

(a)

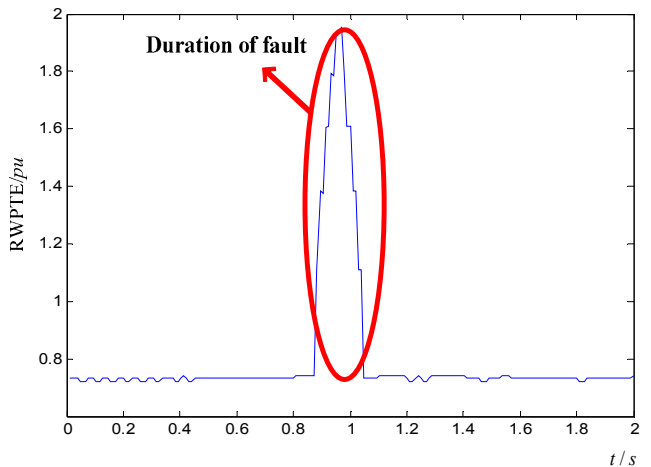

(b)

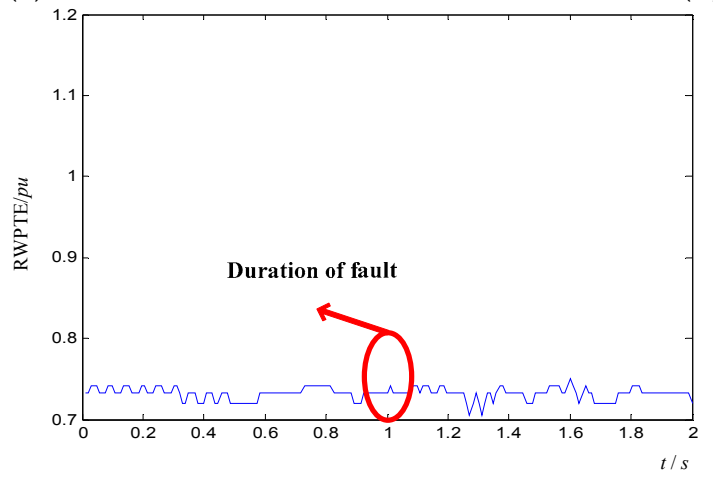

(c)

Figure 12. RWPTE of A, B, C-phase when AB-phase ungrounded short circuit fault occurs. (a) A-phase; (b) B-phase; (c) C-phase. 
When AB-phase grounding short circuit fault and AB-phase ungrounded short circuit fault occur on the AC transmission line, reconstruction signals are computed in 7-14 Hz by RWPTE, and the results are shown in Figure 13. In Figure 13, there are no any significant changes in RWPTE of C-phase when AB-phase ungrounded short circuit fault occurs. Otherwise, AB-phase grounding short circuit fault occurs.

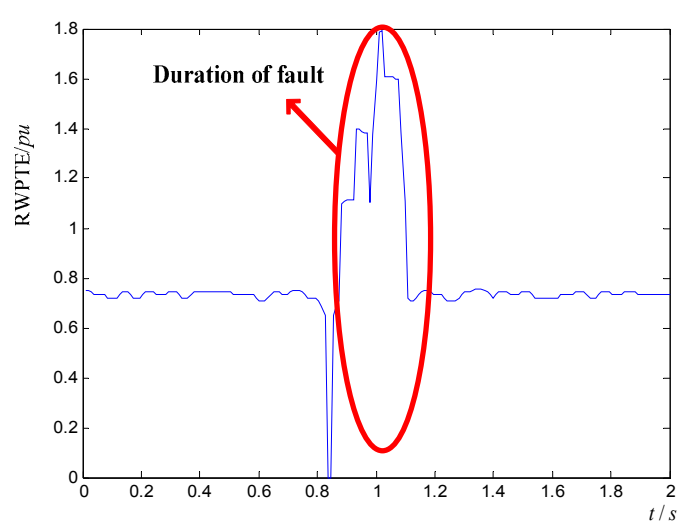

(a)

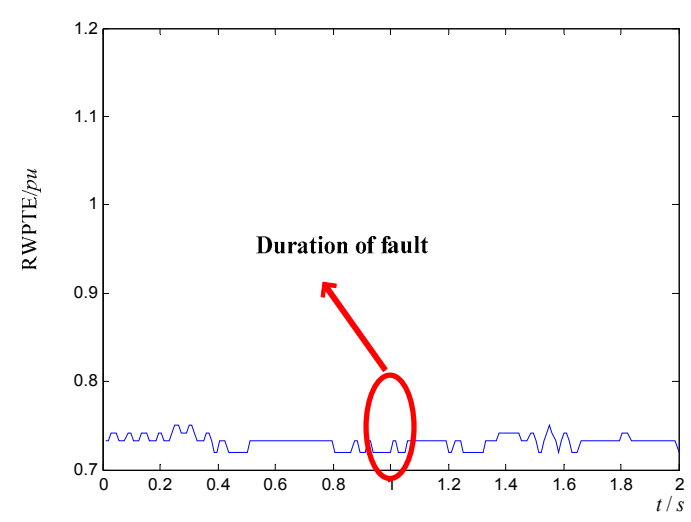

(b)

Figure 13. RWPTE of C-phase. (a) AB-phase grounding short circuit fault; (b) AB-phase ungrounded short circuit fault.

\section{Recognition of AC Transient Fault}

From the Section 4, when the different transient faults occur on AC transmission line, Renyi entropy can be used to extract the fault feature of MMC arms and DC bus when transient faults occur. Based on the feature extraction, a recognition method of AC transient fault is put forward in this article. The flow chart of fault recognition is shown in Figure 14.

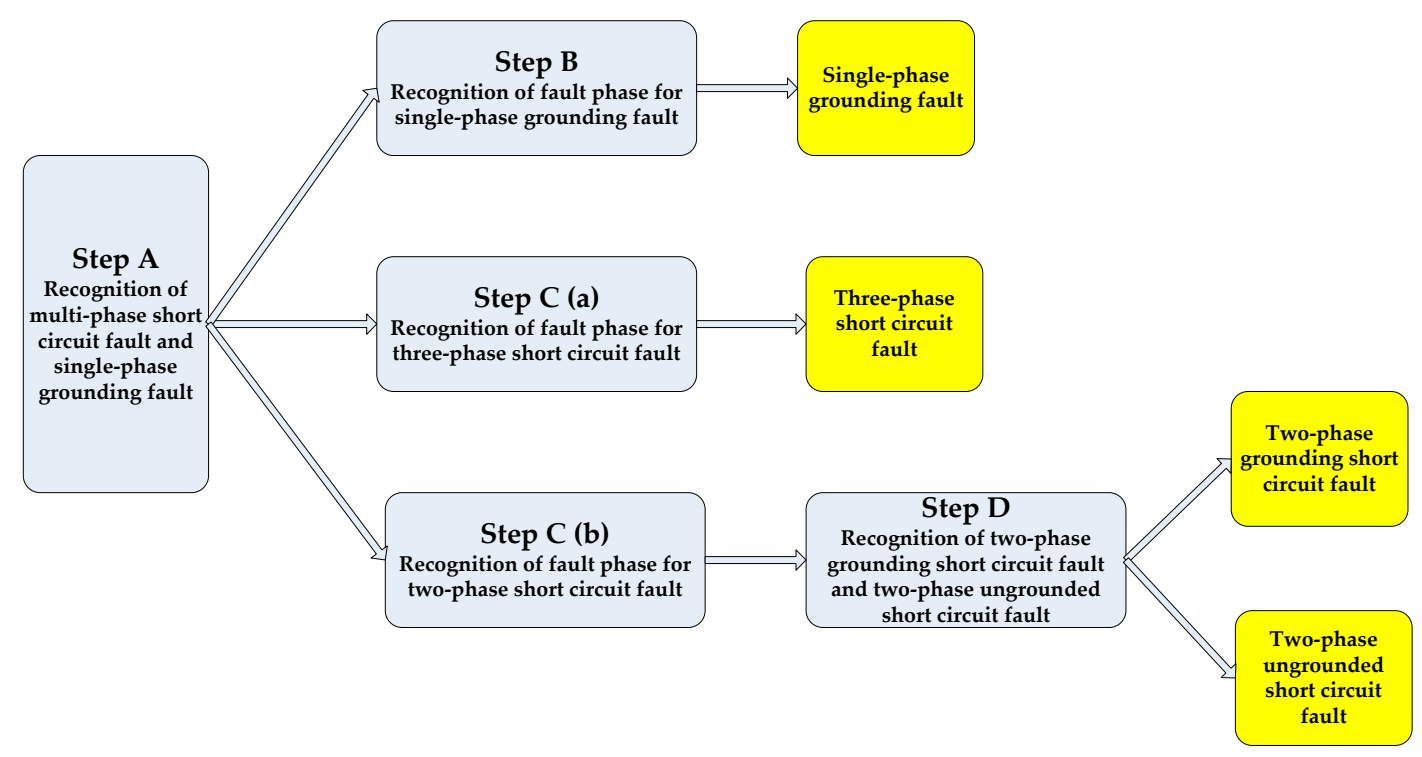

Figure 14. Flow chart of fault recognition.

A. DC bus voltage is sampled with $800 \mathrm{~Hz}$, and RWPEE is performed on it. Comparing the maximum and average value of RWPEE, if the ratio is between 4 and 25, which implies that multi-phase fault occurs on AC transmission line. Otherwise, the single-phase grounding fault occurs. When the single-phase grounding fault occurs, fault phase can be judged by step B. Otherwise, step $\mathbf{C}$ can be used to judge multi-phase fault type and fault phase. 
B. Currents from the upper arm of three phases are respectively sampled with $112 \mathrm{~Hz}$ and RWPTE are computed for reconstruction signal in $42-29 \mathrm{~Hz}$. The phase with entropy saltation is non-fault phase, and the phase without entropy saltation is fault phase. According to it, the fault phase of single-phase grounding fault can be confirmed.

C. Currents from the upper arm of three phases are respectively sampled with $112 \mathrm{~Hz}$ and RWPTE are computed for reconstruction signal in $14-28 \mathrm{~Hz}$.

a. Entropy saltation in three phases implies that three-phase short circuit fault occurs on AC transmission line and the fault phases are $\mathrm{ABC}$ phases.

b. There is two-phase short circuit fault on AC transmission line, when the entropy saltation occurs in only two phases. Although only the two phases with entropy saltation can be determined as fault phase, the other fault phases have to be found based on step $\mathbf{D}$.

D. Fault phases can be identified according to step C. On this basis, RWPTE are computed for reconstruction signal in 7-14 Hz. If there is entropy saltation at the fault phase, it can be determined as two-phase grounding short circuit fault on AC transmission line. Otherwise, it is two-phase ungrounded short circuit fault.

In summary, when transient faults occur on AC transmission line, the steps above can be used to determine the fault type and fault phase.

\section{Stimulation}

In this article, experiment data are acquired by using RT-LAB and MATLAB real-time simulation platform where a MMC-HVDC system model with 2-terminal 51-level is built. A scheme of system model is shown in Figure 15, main parameters are shown in Table 2. Meanwhile, the experiment data are compared with experiment results of MMC-HVDC numeral physics mix platform, which verifies that experiment data can reflect variety law of electrical signals in practical MMC. RT-LAB real-time simulation platform is shown in Figure 16. MMC-HVDC numeral physics mix platform is shown in Figures 17-19.

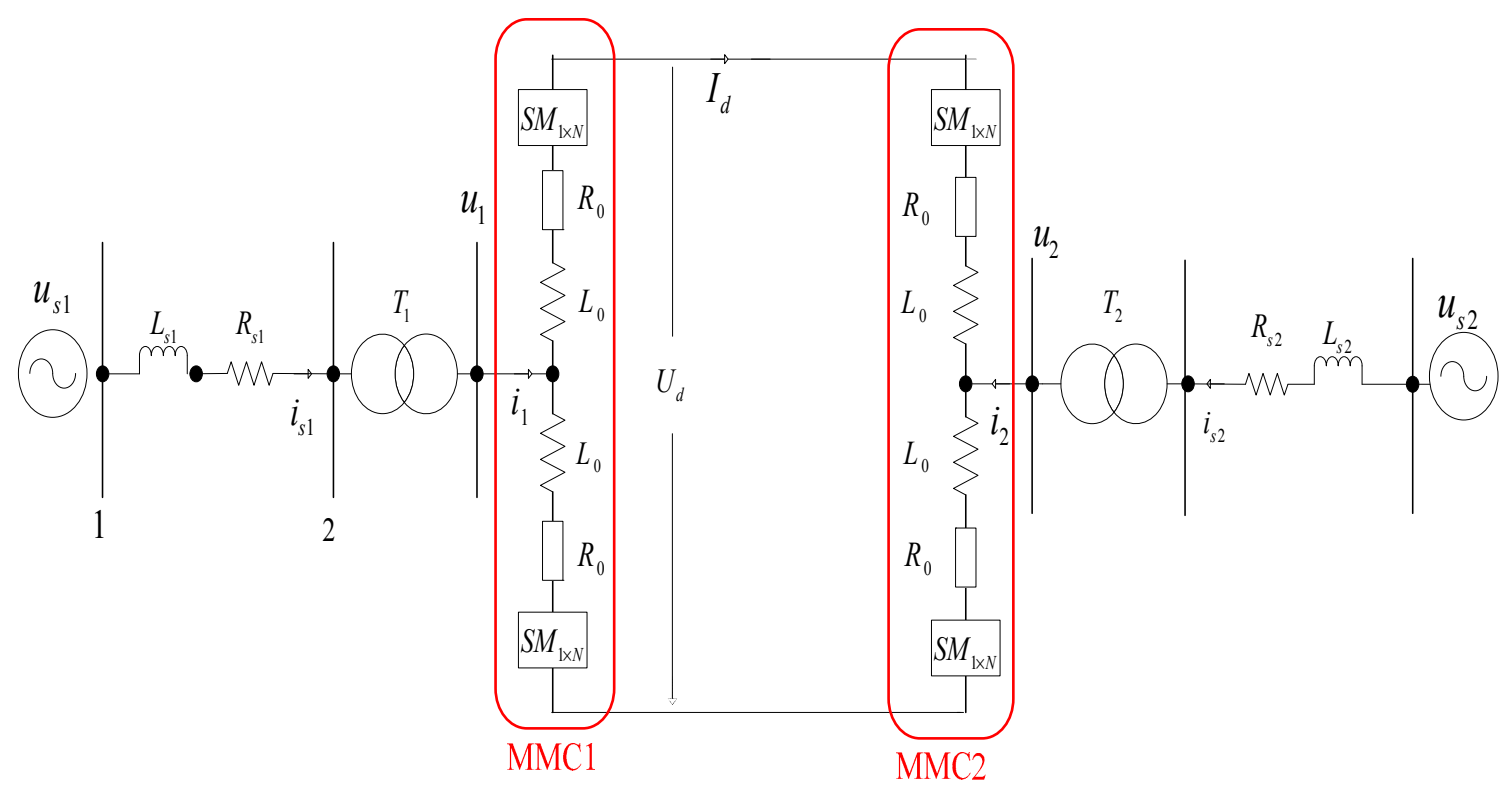

Figure 15. Scheme of system model for 2-terminal 51-level MMC station. 
Table 2. Model parameters.

\begin{tabular}{cc}
\hline Parameters & Value \\
\hline Short circuit ratio & 10 \\
AC system voltage $u_{s 1}, u_{s 2}$ & $230 \mathrm{kV}$ \\
AC system equivalent inductance $L_{s 1}, L_{s 2}$ & $0.2183 \mathrm{mH} / \mathrm{km}$ \\
AC system equivalent resistance $R_{s 1}, R_{s 2}$ & $0.0001143 \Omega / \mathrm{km}$ \\
Transformer turns ratio $u_{s 1} / u_{1}$ & $230 \mathrm{kV} / 64 \mathrm{kV}$ \\
Transformer capacity $T_{1}, T_{2}$ & $230 \mathrm{MVA}$ \\
Connection mode $T_{1}, T_{2}$ & $Y_{0} \mathrm{~d}$ \\
Arm equivalent inductance $L_{0}$ & $24 \mathrm{mH}$ \\
Arm equivalent resistance $R_{0}$ & $0.4 \Omega$ \\
Number of SM & 100 \\
\hline
\end{tabular}

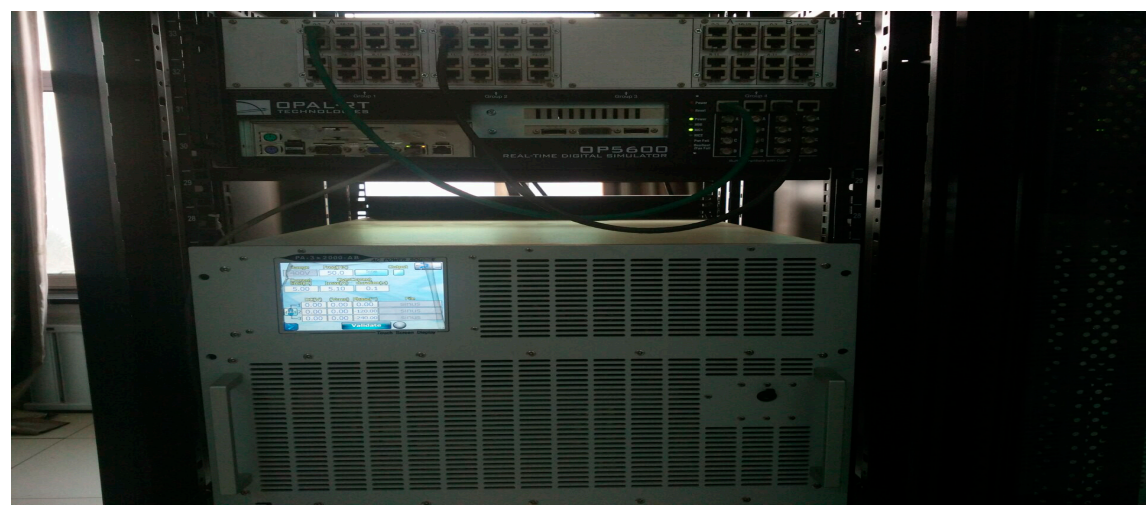

Figure 16. RT-LAB.

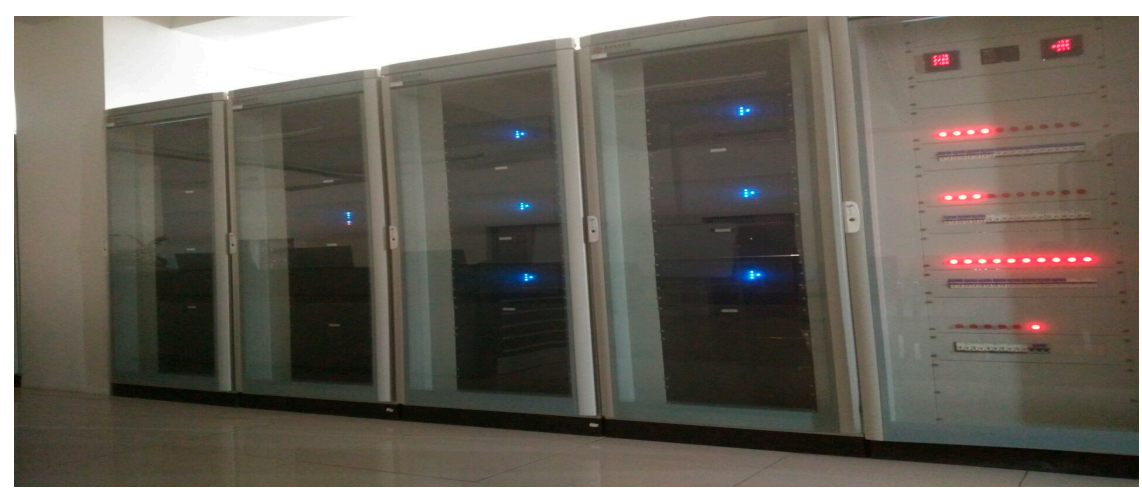

(a)

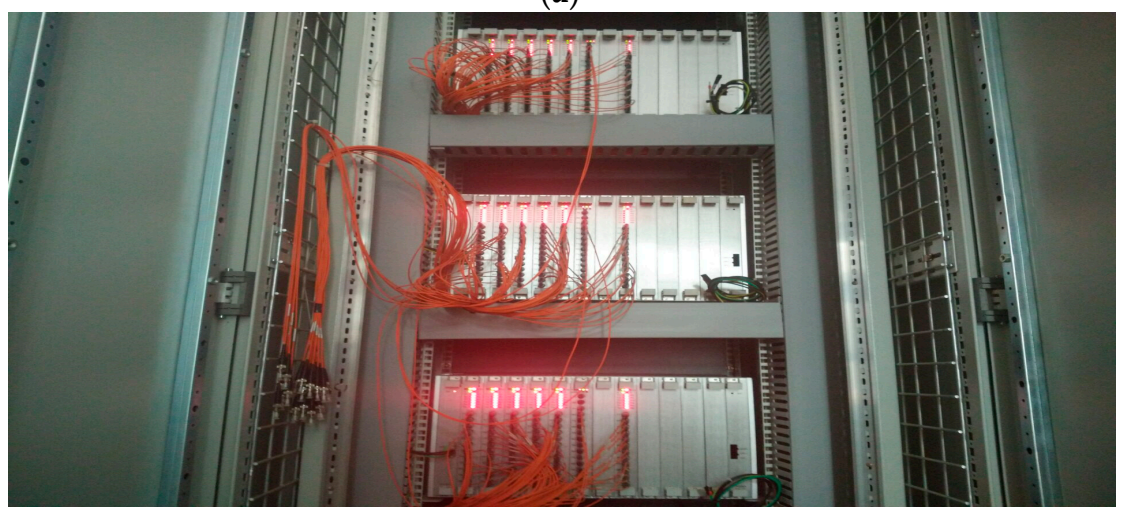

(b)

Figure 17. Valve base controllers. (a) Elevation view; (b) Rear view. 


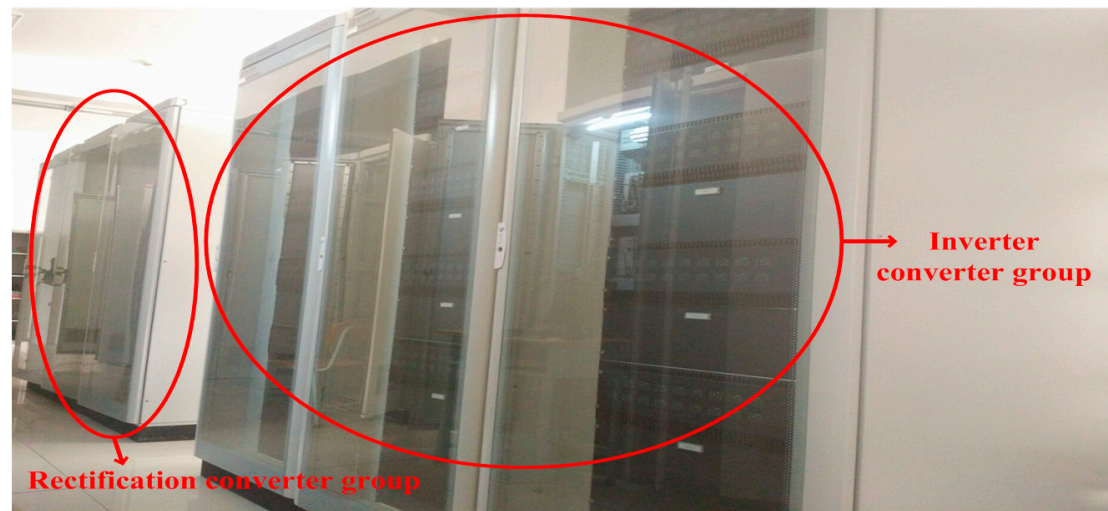

(a)

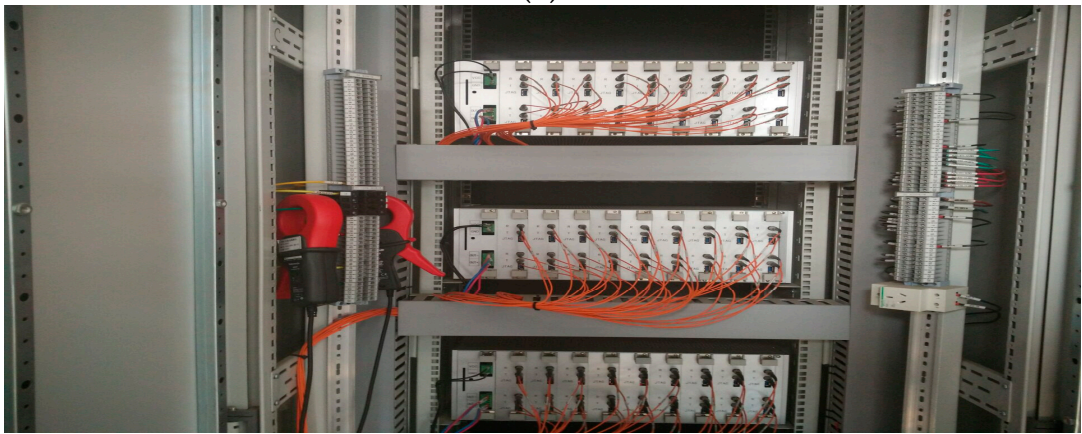

(b)

Figure 18. Converter group. (a) Elevation view; (b) Rear view.

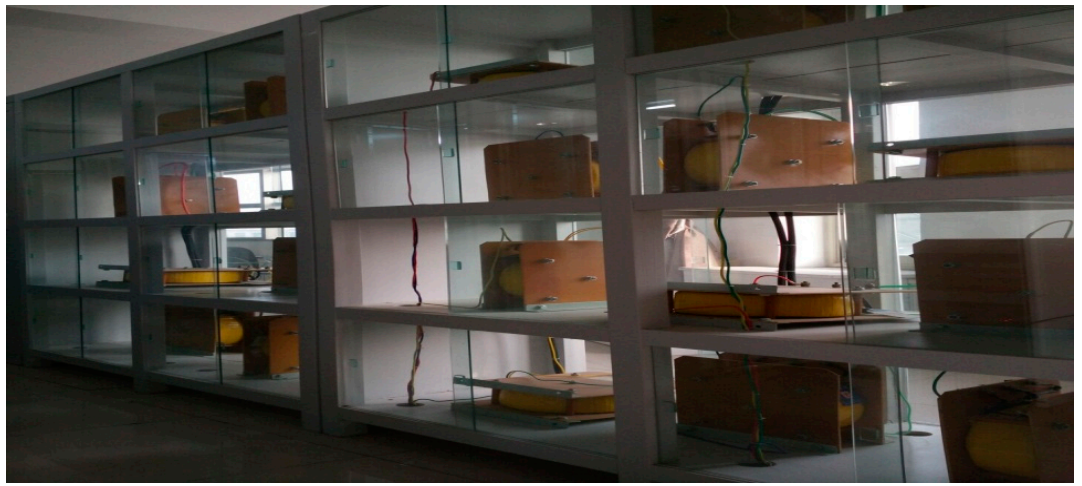

Figure 19. Physic model of DC transmission lines.

Here, the faults are set between 1 and 2 in Figure 15 respectively with grounding resistance of 1-100 $\Omega$. The types of fault include single-phase grounding fault, two-phase short circuit fault, two-phase ungrounded short circuit fault, and three-phase short circuit fault. Each kind of fault occurs 18 times with various grounding resistance and short-circuit resistance.

The feature extraction and recognition method proposed are used to recognize the fault types. The entropy parameter $\alpha$ is 0.1 , the recognition results are shown in Table 3 .

From Table 3, the accuracy rate of fault recognition can reach $93.88 \%$. In addition, the recognition method is compare with back propagation (BP) neural network recognition method that is widely applied to recognize AC fault in power system. The result obtained by BP neural network recognition method is shown in Table 4. From Table 4, the accuracy can reach $90.56 \%$. It is lower than the fault recognition proposed. 
Table 3. The recognition results of transient faults.

\begin{tabular}{cccc}
\hline Fault Types and Fault Phase & $\begin{array}{c}\text { Correct } \\
\text { Recognition }\end{array}$ & $\begin{array}{c}\text { The fault Phase } \\
\text { is Misjudgment }\end{array}$ & $\begin{array}{c}\text { The Fault Type } \\
\text { is Misjudgment }\end{array}$ \\
\hline A-phase groundingfault & 17 & 1 & 0 \\
B-phase groundingfault & 17 & 1 & 0 \\
C-phase groundingfault & 17 & 1 & 0 \\
AB-phase grounding short circuit fault & 17 & 1 & 0 \\
BC-phase grounding short circuit fault & 16 & 1 & 1 \\
AC-phase grounding short circuit fault & 17 & 0 & 1 \\
AB-phase ungrounded short circuit fault & 17 & 1 & 0 \\
BC-phase ungrounded short circuit fault & 16 & 1 & 1 \\
AC-phase ungrounded short circuit fault & 17 & 0 & 0 \\
ABC-phase short circuit fault & 18 & & 0 \\
\hline
\end{tabular}

Table 4. The results of BP neural network recognition method.

\begin{tabular}{cccc}
\hline Fault Types and Fault Phase & $\begin{array}{c}\text { Correct } \\
\text { Recognition }\end{array}$ & $\begin{array}{c}\text { The Fault Phase } \\
\text { is Misjudgment }\end{array}$ & $\begin{array}{c}\text { The Fault Type } \\
\text { is Misjudgment }\end{array}$ \\
\hline A-phase grounding fault & 16 & 1 & 1 \\
B-phase grounding fault & 17 & 1 & 0 \\
C-phase grounding fault & 16 & 2 & 0 \\
AB-phase grounding short circuit fault & 16 & 1 & 1 \\
BC-phase grounding short circuit fault & 15 & 2 & 1 \\
AC-phase grounding short circuit fault & 16 & 1 & 1 \\
AB-phase ungrounded short circuit fault & 16 & 1 & 0 \\
BC-phase ungrounded short circuit fault & 16 & 2 & 0 \\
AC-phase ungrounded short circuit fault & 17 & 1 & 0 \\
ABC-phase short circuit fault & 18 & 0 & \\
\hline
\end{tabular}

\section{Conclusions}

In this article, the mechanism and feature of transient faults is analyzed for two-terminal MMC-HVDC system and the mathematical expression is established. Based on the characteristics of electrical signals in MMC as AC transient faults occur, RWPEE and RWPTE are proposed to extract feature of transient faults. Combining the results of feature extraction, a novel recognition method is proposed to recognize AC transient faults and its effectiveness is verified. The conclusions are as follows.

(a) Different AC transient faults have different influence on MMC. It is difficult to recognize AC transient faults by using time-domain signals of electrical information in MMC.

(b) Based on high resolution of DWPT in frequency-domain, the energy distribution of electrical signals in MMC are different for different arms in the same frequency bands after DWPT. According to the feature of energy distribution in the same frequency bands, RWPEE and RWPTE are proposed to extract feature of transient faults. In term of feature extraction accuracy and computation complexity, the methods proposed are better than SWPEE and SWPTE.

(c) The recognition method proposed can simplify numerous and complicated electrical information and it achieves the recognition of AC transient faults in MMC-HVDC system. Compared with BP neural network recognition, the recognition method proposed is more accurate.

(d) The conclusion provides theoretical support for the further analysis of operation state and AC-DC interaction for MMC-HVDC system. The next research will focus on how to reduce negative effects of transient faults on MMC from the results of this article, and the control strategy and evaluation of operation state will be proposed to optimize the performance of MMC-HVDC system. 
Acknowledgments: The financial support received from the Project Supported by the Science and Technology Development Plan of State Grid, China (Grant No. NEDU20150002) is gratefully acknowledged.

Author Contributions: Jikai Chen conceived the idea, Yanhui Dou, Yang Li and Jiang Li performed experiments and data analysis, and Jikai Chen, Yanhui Dou, Yang Li, Jiang Li and Guoqing Li wrote the article. All authors have read and approved the final manuscript.

Conflicts of Interest: The authors declare no conflict of interest.

\section{References}

1. Liu, H.; Ma, K.; Loh, P.C.; Blaabjerg, F. Online Fault Identification Based on an Adaptive Observer for Modular Multilevel Converters Applied to Wind Power Generation Systems. Energies 2015, 8, 7140-7160. [CrossRef]

2. Saad, H.; Mahseredjian, J.; Dennetiere, S.; Nguefeu, S. Interactions studies of HVDC-MMC link embedded in an AC grid. Electr. Power Syst. Res. 2016, 138, 202-209. [CrossRef]

3. Tu, Q.; Xu, Z. Impact of Sampling Frequency on Harmonic Distortion for Modular Multilevel Converter. IEEE Trans. Power Del. 2011, 26, 298-306. [CrossRef]

4. Yu, F.; Wang, X.; Zhang, Y.; Wang, Z. Direct Circulating Current Suppressing Strategy for MMC under Unbalanced AC Grid Conditions. Power Syst. Technol. 2015, 39, 3339-3345.

5. Hu, P.; Jiang, D.; Zhou, Y.; Liang, Y.; Guo, J.; Lin, Z. Energy-balancing control strategy for modular multilevel converters under sub-module fault conditions. IEEE Trans. Power Electron. 2014, 29, 5021-5030. [CrossRef]

6. Tang, G.; Xu, Z.; Zhou, Y. Control and modulation strategies for modular multilevel converter based HDVC system. Power Syst. 2014, 29, 3030-3040. [CrossRef]

7. Zhang, J.; Zhao, C. Control Strategy of MMC-HVDC under Unbalanced Grid Voltage Conditions. J. Power Electron. 2015, 15, 1499-1507. [CrossRef]

8. Liu, Y.; Chen, Q.; Li, N.; Xie, B.; Wang, J.; Ji, Y. On DC-Side Impedance Frequency Characteristics Analysis and DC Voltage Ripple Prediction under Unbalanced Conditions for MMC-HVDC System Based on Maximum Modulation Index. J. Power Electron. 2016, 16, 319-328. [CrossRef]

9. Ludois, D.; Venkataramanan, G. An Examination of AC/HVDC Power Circuits for Interconnecting Bulk Wind Generation with the Electric Grid. Energies 2010, 3, 1263-1289. [CrossRef]

10. Xu, C.; Dai, K.; Chen, X.; Kang, Y. Voltage droopcontrolat point of common coupling with arm current and capacitor voltage analysis for distribution static synchronous compensator based on modular multilevel converter. IET Power Electron. 2016, 9, 1643-1653. [CrossRef]

11. Guan, M.; Xu, Z.; Tu, Q.; Pan, W. Nearest Level Modulation for Modular Multilevel Converters in HVDC Transmission. Autom. Electr. Power Syst. 2010, 34, 48-52.

12. Shao, S.; Wheeler, P.W.; Clare, J.C.; Watson, A.J. Fault detection for modular multilevel converters based on sliding mode observer. IEEE Trans. Power Electron. 2013, 28, 4867-4872. [CrossRef]

13. Ma, Y.; Wang, W.; Zhang, J.; Tang, J.; Ren, T. Analysis of MMC-HVDC Transient Response Characteristic under Typical Disturbances. Proc. CSU-EPSA 2011, 23, 110-118.

14. Qiu, P.; Zhang, S.; Huang, X.; Lu, Y.; Lu, M. Research on the protection strategies of internal AC bus fault based on MMC-HVDC. Power Syst. Prot. Control 2014, 42, 150-154.

15. Ma, S.; Yu, L.; Zheng, L. Characteristics Analysis of MMC-HVDC AC Asymmetric Fault and Protection Strategy. J. North China Electr. Power Univ. 2015, 42, 35-43.

16. Xiao, L.; Xu, Z.; Liu, S.; An, T.; Kong, M. AC Fault Ride through Strategy of MMC-HVDC Connected to Passive Networks. Trans. China Electrotech. Soc. 2016, 31, 120-128.

17. Liang, Y.; Zhang, T.; Liu, J.; Yang, Q.; Liu, S. A Circulating Current Suppressing Method for Modular Multilevel Converter under Unbalanced Grid Voltage. Trans. China Electrotech. Soc. 2016, 31, 120-128.

18. Yuan, X.; Gao, L.; Wen, J.; Cheng, S. Unbalanced three-phase control strategy of VSC-HVDC system. Electr. Power Autom. Equip. 2010, 30, 1-5.

19. Li, X.; Liu, W.; Song, Q.; Rao, H.; Zhu, Z.; Li, X. An Enhanced MMC Topology with DC Fault Clearance Capability. Proc. CSEE 2011, 34, 6389-6397.

20. Li, X.; Song, Q.; Liu, W.; Li, Q.; Rao, H.; Xu, S. Zero-sequence voltage injection control scheme of modular multilevel converter supplying passive networks under unbalanced load conditions. Electr. Power Syst. Res. 2015, 121, 270-278. [CrossRef] 
21. Guan, M.; Xu, Z. Modeling and control of a modular multilevel converter-based HVDC system under unbalanced grid conditions. IEEE Trans. Power Electron. 2012, 27, 4858-4867. [CrossRef]

22. Guan, M.; Xu, Z.; Pan, W.; Zhang, J.; Zheng, X. Analysis and Control of Modular Multilevel Converter Based HVDC Transmission Systems during Grid Faults. High Volt. Eng. 2013, 39, 1238-1245.

23. Liu, Z.; Cui, Y.; Li, W. Combined Power Quality Disturbances Recognition Using Wavelet Packet Entropies and S-Transform. Entropy 2015, 17, 5811-5828. [CrossRef]

24. Chen, J.; Zhou, Z.; Li, H.; Xu, B. Study of Fast Wavelet Entropy's Application in Feature Extraction of Transient Signals in Transmission Line. Trans. China Electrotech. Soc. 2012, 27, 219-225.

25. Andreopoulos, Y.; Schaar, M.V.D. Generalized Phase Shifting for M-Band Discrete Wavelet Packet Transforms. IEEE Trans. Signal Proc. 2007, 52, 742-747. [CrossRef]

26. Erdogmus, D.; Hild, K.E.; Principe, J.C.; Lazaro, M.; Santamaria, I. Adaptive blind deconvolution of linear channels using Renyi's entropy with Parzen window estimation. IEEE Trans. Signal Proc. 2014, 52, 1489-1498. [CrossRef]

27. Chen, J.; Dou, Y.; Wang, Z.; Li, G. A Novel Method for PD Feature Extraction of Power Cable with Renyi Entropy. Entropy 2015, 17, 7698-7712. [CrossRef]

28. Chen, J.; Li, H.Y.; Yang, S.; Kou, B. Application of Wavelet Packet Singularity Entropy and PSD in Power Harmonics Detection. Trans. China Electrotech. Soc. 2010, 25, 193-199.

29. Sun, L.; Hu, X.; Ji, Y. Fault Diagnosis for High Voltage Circuit Breakers with Improved Characteristic Entropy of Wavelet Packet. Proc. CSEE 2007, 27, 103-108.

30. Chen, J.; Li, G. Tsallis Wavelet Entropy and Its Application in Power Signal Analysis. Entropy 2014, 16, 3009-3025. [CrossRef]

(C) 2016 by the authors; licensee MDPI, Basel, Switzerland. This article is an open access article distributed under the terms and conditions of the Creative Commons Attribution (CC-BY) license (http:/ / creativecommons.org/licenses/by/4.0/). 